\title{
Multi-generational analysis and visualization of the vascular tree in 3D micro-CT images
}

\author{
Shu-Yen Wan ${ }^{\mathrm{a}}$, Erik L. Ritman ${ }^{\mathrm{b}}$, William E. Higgins ${ }^{\mathrm{c}, *}$ \\ ${ }^{a}$ Department of Computer Science and Information Engineering, Chang Gung University, Taiwan, ROC \\ ${ }^{\mathrm{b}}$ Department of Physiology and Biophysics, Mayo Clinic, Rochester, MN, USA \\ ${ }^{c}$ Department of Electrical Engineering, Pennsylvania State University, University Park, PA 16802, USA
}

Received 19 July 2001

\begin{abstract}
Micro-CT scanners can generate large high-resolution three-dimensional (3D) digital images of small-animal organs, such as rat hearts. Such images enable studies of basic physiologic questions on coronary branching geometry and fluid transport. Performing such an analysis requires three steps: (1) extract the arterial tree from the image; (2) compute quantitative geometric data from the extracted tree; and (3) perform a numerical analysis of the computed data. Because a typical coronary arterial tree consists of hundreds of branches and many generations, it is impractical to perform such an integrated study manually. An automatic method exists for performing step (1), extracting the tree, but little effort has been made on the other two steps. We propose an environment for performing a complete study. Quantitative measures for arterial-lumen cross-sectional area, inter-branch segment length, branch surface area and others at the generation, inter-branch, and intra-branch levels are computed. A human user can then work with the quantitative data in an interactive visualization system. The system provides various forms of viewing and permits interactive tree editing for "on the fly" correction of the quantitative data. We illustrate the methodology for 3D micro-CT rat heart images. (C) 2002 Elsevier Science Ltd. All rights reserved.
\end{abstract}

Keywords: Micro-CT imaging; 3D vascular tree analysis; Coronary arteries; Small-animal imaging; Volume visualization; 3D medical image processing

\section{Introduction}

Modern micro-CT scanners can produce very large three-dimensional (3D) digital images of vascular trees [1-4]. A typical tree consists of hundreds of branches and many generations. The spatial

${ }^{*}$ Corresponding author. Tel.: +1-814-865-0186; fax: +1-814-863-5341.

E-mail addresses: sywan@mail.cgu.edu.tw (S.-Y. Wan), elran@mayo.edu (E. L. Ritman),weh2@psu.edu (W. E. Higgins). 
resolution of 3D micro-CT images is fine enough to permit detailed real studies of basic physiologic questions on the entire coronary arterial tree, from aorta to terminal arterioles. For example, previously proposed mathematical laws governing the branching geometry and fluid transport mechanisms of the coronary arterial tree can be studied [5-10]. 3D micro-CT images of hearts from rats can now be conceivably undertaken. Performing a study requires three steps:

(1) Extract the arterial tree from the image.

(2) Compute quantitative data from the extracted tree.

(3) Do a numerical analysis of the computed tree geometry.

The segmentation, visualization, and analysis of a complete tree is a major logistics problem. In fact, most previously completed studies focused either on extracting one complete principal pathway through a tree or analyzed traced branch segments $[2,11,12]$. These studies used painstaking manual image analysis for the extraction. It is unclear, however, that such analyses fairly represents the structure and function of the entire tree. The complexity and sheer size of a tree makes it impractical to use purely manual image analysis for extracting an entire tree.

A recent effort proposed an approach for extracting the entire tree automatically [4]. This effort, however, does not consider the subsequent quantitative and numerical analyses (steps 2-3 above). We propose an environment for performing these analyses. A set of quantitative measures are computed from the extracted tree data. These measures form part of a tree representation scheme for subsequent analysis. A graphical user interface (GUI) system enables the user to interact with the representation. The interactive system provides an intuitive means for understanding the detailed statistical structure of the tree. The results show that multi-generational analysis of large branching trees is feasible, but, for the domain of micro-CT imaging, further work is needed in improving the data and in building more complete analysis methods.

\section{Specimen preparation and image processing}

This section overviews the procedures for specimen preparation, micro-CT scanning, and subsequent tree extraction (Step 1). All of these aspects of the experimental methods have been described in greater detail elsewhere $[2,4]$.

\subsection{Specimen preparation}

Sprague Dawley rats were used for this study. The rats were anesthetized by an intra-peritoneal injection of $35 \mathrm{mg} / \mathrm{kg}$ pentobarbital sodium and the aorta canulated. Next, the rats were euthanized by a $20 \mathrm{mEq}$ intravenous injection of KCI solution. Heparinized saline $\left(5000 \mathrm{units} / 100 \mathrm{~cm}^{3}\right)$ and a vasodilator (Lidocaine, $1 \mathrm{mg} / \mathrm{kg}$ ) were used to flush the vascular system, followed by infusion of microfil (a silicon polymer containing lead chromate) at $100 \mathrm{~mm} \mathrm{Hg}$ pressure using a Harvard pump. As soon as the microfil entered both venaecava and pulmonary artery, the pulmonary artery and aorta were clamped and the infusion was stopped. During the $20 \mathrm{~min}$ it took the microfil to polymerize, the intravascular pressure dropped, generally to about $60 \mathrm{~mm} \mathrm{Hg}$. Next, the hearts were dissected free and immersed in 10\% formalin for 3 days following which they were progressively dehydrated with increasing concentrations of glycerol. Finally, each specimen was embedded in a closely fitting cylindrical container filled with bioplastic. Occasionally, the microfil in the vascular 


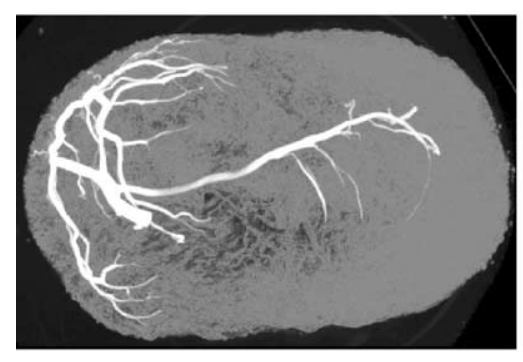

(a) Transverse

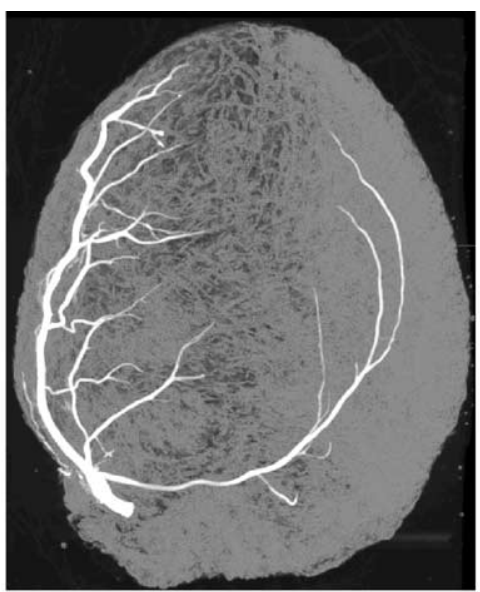

(b) Coronal

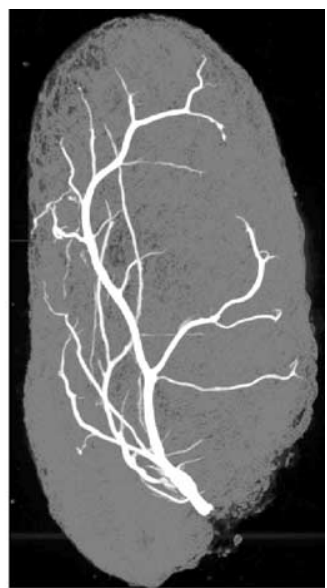

(c) Sagittal

Fig. 1. Maximum intensity projections (MIP) of a micro-CT volume of a rat's left circumflex arterial tree ("lca_146"). (a) 3D-to-2D projection in the transverse direction ( $x-y$ plane); (b) coronal projection ( $x-z$ plane); (c) sagittal projection $(y-z$ plane $)$. The arterial tree is the bright structure. The image dimensions are $401 \times 267 \times 490$ cubic voxels, and the voxel size is $20.8 \mu \mathrm{m}^{3}$.

lumen contains gas bubbles or is kept from fully filling a vessel lumen by a thrombus that was not fully flushed out. In addition $\mathrm{PbO}_{4}$ contained in the microfil can segregate into clumps.

\subsection{Micro-CT scanner}

A prepared specimen was placed on top of a computer-controlled rotation stage in the micro-CT scanner. The scanner is described in detail by Jorgensen et al. [2]. We describe here only the details directly relevant to this study. In brief, it consists of a spectroscopy X-ray source that generates $\mathrm{X}$-ray photons of a nominal $18 \mathrm{keV}$ energy. The X-rays transmitted through the specimen expose a crystalline plate of cesium iodide, doped with thallium, which converts the X-ray image to a light image. This image is focused onto a CCD detector array $(1024 \times 1024$ square pixels, each $20.8 \mu \mathrm{m}$ on a side) with a microscope objective operated at $1: 1$ or $1: 4$ magnifications. The specimen is rotated in $0.5^{\circ}$ steps around $360^{\circ}$ about its vertical axis in between each X-ray exposure. The recorded X-ray projection gray scale was then converted to logarithmic values and a modified Feldkamp cone beam tomographic reconstruction performed. The resulting 3D image has cubic voxels; i.e., the real sample spacing is equal along all three Cartesian axes $x, y$, and $z: \Delta x=\Delta y=\Delta z=\Delta$. Each voxel has 16 bits of gray-scale information. Quantum wobble results in gray-scale noise and occasional "cosmic rays" cause localized bright spots in random locations. Fig. 1 shows an example image.

\subsection{Tree extraction}

After obtaining a 3D micro-CT rat heart image, we apply an image processing procedure that extracts the vascular tree and generates a skeleton representation; see Fig. 2. The processing steps 


\section{EXTRACTION OF TREE}

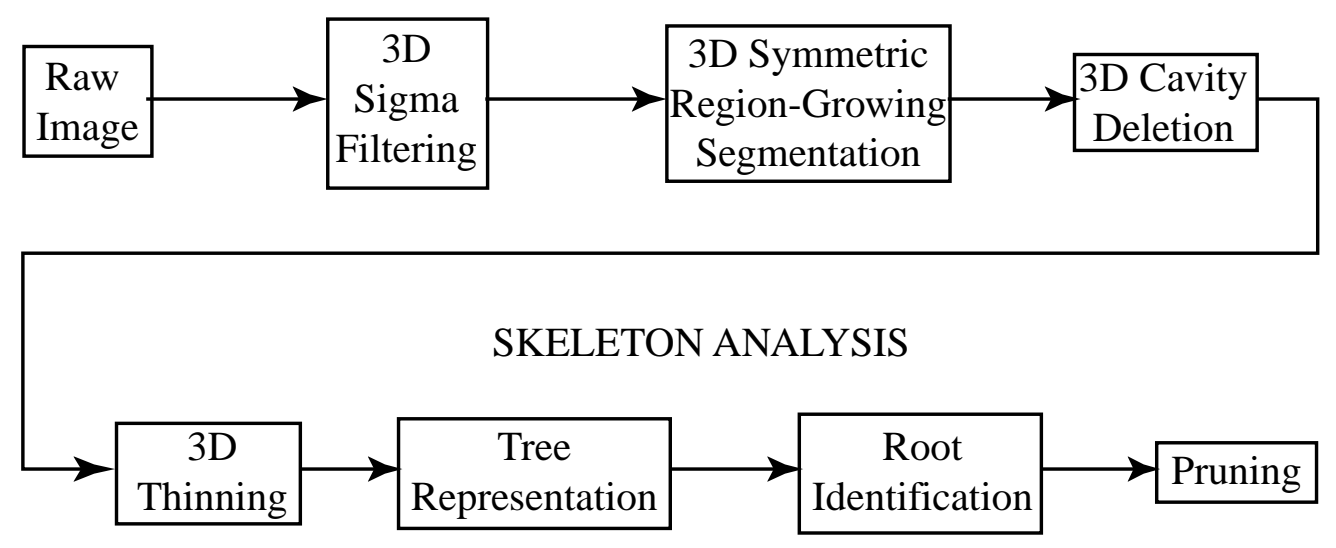

Fig. 2. Image processing procedure.

include:

(1) 3D sigma filtering, to suppress noise while preserving thin structures [13],

(2) 3D symmetric region growing segmentation, to extract the desired raw tree [14],

(3) 3D cavity deletion, to fill possible 3D holes inside the tree branches and produce a solid tree.

(4) 3D thinning, to define the skeleton of the tree while preserving homotopy [15],

(5) tree representation, to convert the image-form tree skeleton data into a compact graph-like data structure [16],

(6) root identification, to help put the tree's branching geometry in proper hierarchical order.

(7) pruning, to remove unwanted branches or extraneous trees.

The main tree root (not used until step 6) is specified interactively before the procedure is begun. All subsequent processing is fully automatic and 3D. 3D sigma filtering removes high-frequency noise and preserves thin structures. After 3D symmetric region-growing segmentation and 3D cavity deletion, a raw segmented tree exists. 3D thinning produces an approximate central-axes representation of the tree. The tree representation step converts the image-form of the skeleton into a much more compact data structure. The data structure captures the complete axial structure of the tree branches. It also includes mother/child/sibling relationships for the tree branches. The user manually specifies the root of the tree for root identification. Given the root, the tree representation is reorganized to give the complete correct tree hierarchy. The raw tree tends to have some extraneous noise branches; the final pruning step removes these extraneous branches. Ref. [4] describes the procedure fully.

The final outputs of the procedure are the segmented arterial tree and the tree representation. Fig. 3 depicts example results for the procedure. The procedure has been validated for a number of micro-CT images and compared to manually defined ground truth data [4]. A limitation of these results, however, is that, for a given $3 \mathrm{D}$ image, validation could only be done for one complete branch. This is because it was not feasible to define more than one complete branch manually. (Phantom results, however, did consider a whole tree [4], but these were done under idealized 


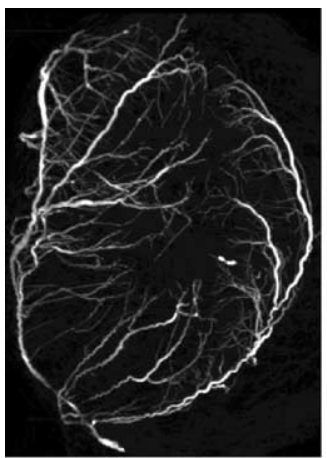

(a) Original

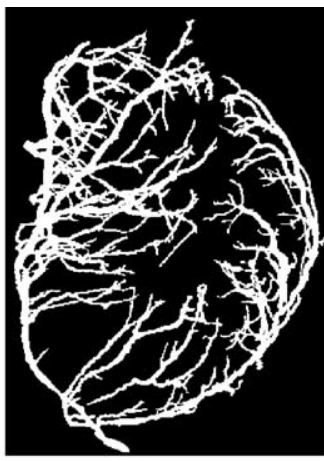

(b) Segmented tree

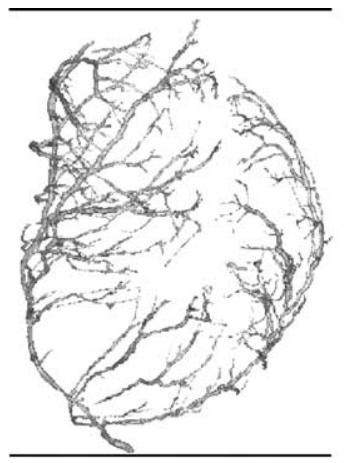

(c) Surface rendering

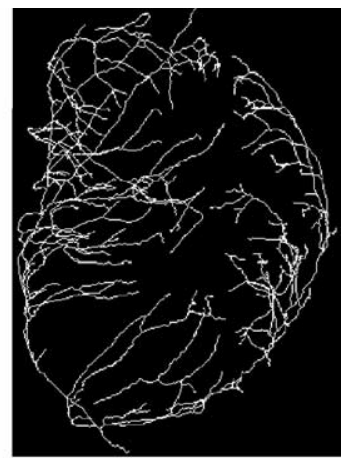

(d) Skeleton

Fig. 3. Results of applying the analysis procedure of Fig. 4 to a rat's left circumflex arterial image ("Ica_40"). All images except (c) are maximum-intensity projections of the 3D data. The original lca_40 image, as shown in (a), has voxel resolution of $20.7 \mu \mathrm{m}$ in each direction, $x, y$, and $z$; i.e., $\Delta x=\Delta y=\Delta z=20.7 \mu \mathrm{m}$. The image's dimensions are $381 \times 336 \times 455$. For $3 \mathrm{D}$ sigma filtering, the windowing operator has size $3 \times 3 \times 3$ and $\sigma=10$. The segmentation, shown in part (b), was generated with the following parameters: $G_{\min }=40 ; G_{\text {seed_min }}=90 ; G_{\text {seed_max }}=G_{\max }=255 ; G_{\text {tolerance }}=10$; $G_{\text {size_min }}=100 ; G_{\text {size_seed }}=10 ; G_{\text {connectivity }}=26$ [4]. Part (c) shows a surface-rendered view of the segmentation; it was made using the interactive system discussed in Section 3. The skeleton (d) shows the complete set of extracted central axes.

conditions.) While these results give considerable confidence that the method gives useful "raw" results, it does not provide insight into the efficacy of subsequent multi-generational analysis of the entire tree. It is our purpose now to address this issue.

\section{Quantitation and visualization of multi-generational analyses}

The segmented arterial tree and its associated skeletal structure are used to compute a series of measures that quantify the structure of the tree. This section describes the quantitative measures computed for a tree (Section 3.1) and the proposed interactive tool for working with the quantitative data (Section 3.2).

\subsection{Quantitation}

Many measurements can be made on the extracted tree data to quantitate its inherent geometrical information. Before proceeding, we first make a few definitions. A branch is defined as a section of an artery terminated by either (1) an endpoint and a bifurcation point or by (2) two sequential bifurcation points. The tree begins with the root branch, which is denoted as generation 0 . The root branch bifurcates to form two daughter branches at generation 1, etc. All of these definitions are made relative to the skeletal structure. See Fig. 4 for an example. Now, consider the following general mathematical quantities:

$g_{i}$ ith generation,

$b_{j} j$ th branch, 


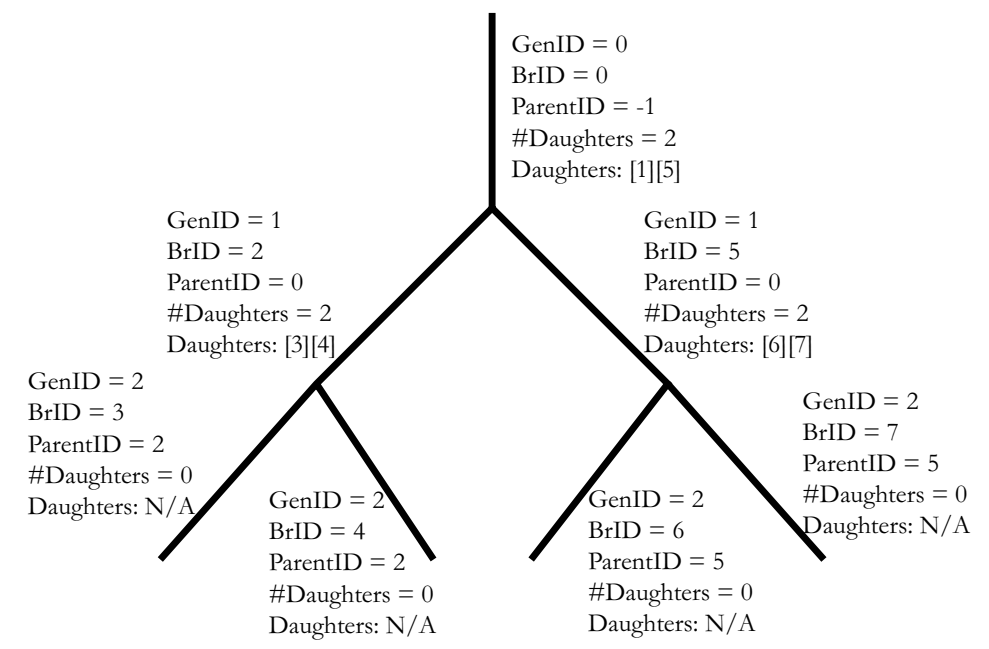

Fig. 4. Example of a tree skeletal structure for a tree containing two generations of branches. Each branch has associated with it information conveying the hierarchical properties of the branch. "GenID" denotes generation ID; "BrID" denotes branch ID; "ParentID" denotes parent ID; “\# Daughter" represents the number of daughters; "Daughters" lists the branch IDs of daughters.

$c_{j, k} k$ th cross-section of branch $b_{j}$,

$N^{\mathrm{b}}(i)$ number of branches in generation $g_{i}$,

$N^{\mathrm{c}}(j)$ number of cross-sections (or sites) constituting branch $b_{j}$,

$p_{i}$ starting ID for branches of generation $g_{i}$.

We approximate each tree branch $b_{j}$ 's central axis by a set of connected line segments. The line segments are computed by minimizing the difference between the original vasculature and curve-fitted segments, as described elsewhere $[17,18]$. The line segments in turn are represented as a series of 3D discrete sites, $k=1,2, \ldots, N^{\mathrm{c}}(j)$, that are one voxel apart. Thus, adjacent sites can be a Euclidean distance $1, \sqrt{2}$, or $\sqrt{3}$ apart. A $2 \mathrm{D}$ cross-section $c_{j, k}$, consisting of voxels in the segmented tree, is defined perpendicular to each branch site $k$. Next, a series of measurements is made for each branch, as listed below:

$L\left(b_{j}\right)$ length of branch $b_{j}$,

$R\left(b_{j}\right)$ distance from $b_{j}$ to the root,

$D\left(b_{j}\right)$ average diameter of a cross-section in $b_{j}$,

$A\left(c_{j, k}\right)$ cross-sectional area (CSA) of $c_{j, k}$,

$L D\left(b_{j}\right)$ average $\log -\log$ ratio between branch length and diameter for $b_{j}=\log L\left(b_{j}\right) / \log D\left(b_{j}\right)$,

$S\left(b_{j}\right)$ surface area of $b_{j}$,

$V\left(b_{j}\right)$ volume of $b_{j}$,

$V_{\text {loss }}\left(b_{j}\right)$ volume loss of $b_{j}$ as defined by Eq. (4).

All length, distance, and other measures are made using Euclidean digital distance and real voxel spacing $4 . R\left(b_{j}\right)$ is computed by traversing the distance travelled from the first site of $b_{j}$ to the root. A cross-section $c_{j, k}$ 's diameter is defined as the length of $c_{j, k}$ 's major axis. $D\left(b_{j}\right)$ is the average of these values for $b_{j} . A\left(c_{j, k}\right)$, the CSA of cross-section $c_{j, k}$, is defined as the number of voxels in $c_{j, k}$ 
times $\Delta^{2}$. Note that a branch cross-section is ill-defined at a bifurcation, because of the nature of a branching tree's geometry near a bifurcation. Thus, we do not use CSA measures for cross-sections adjacent to bifurcation points. The average CSA of a cross-section in branch $b_{j}$ is given by

$$
\mu_{A}\left(b_{j}\right)=\frac{\sum_{k=2}^{N^{\mathrm{c}}(j)-1} A\left(c_{j, k}\right)}{N^{\mathrm{c}}(j)} .
$$

The CSA values along branches are employed to give measures of intra- or inter-generation data on surface areas, volumes, surface variations, and other measurements [19]. To compute the surface area of a branch $b_{j}$, we first assume that each cross-section $c_{j, k}$ having CSA $A\left(c_{j, k}\right)$ approximates a circular disk. Since a circular disk has area $\pi r^{2}$, then, letting $A\left(c_{j, k}\right) \approx \pi r_{j, k}^{2}$ gives $r_{j, k}=\sqrt{A\left(c_{j, k}\right) / \pi}$. The surface area contribution of cross-section $c_{j, k}$ then is $2 \pi r_{j, k}=2 \sqrt{\pi A\left(c_{j, k}\right)}$. Thus, the total surface area of branch $b_{j}$ is given by

$$
S\left(b_{j}\right)=\sum_{k=1}^{N^{\mathrm{c}}(j)} 2 \Delta_{k} \sqrt{\pi A\left(c_{j, k}\right)},
$$

where $\Delta_{k}$ gives the distance between sites $k-1$ and $k\left(\Delta_{k}=\Delta \times 1, \sqrt{2}\right.$, or $\left.\sqrt{3}\right)$. Eq. (2) assumes that a branch can be approximated as contiguous series of thin "cylinders" (each cross-section $c_{j, k}$ contributes a cylindrical disk of thickness $\Delta_{k}$ ). Similarly, the volume of a branch $b_{j}$ is given by the sum of CSAs of $b_{j}$ 's cross-sections weighted by the cross-section spacing:

$$
V\left(b_{j}\right)=\sum_{k=1}^{N^{\mathrm{c}}(j)} \Delta_{k} A\left(c_{j, k}\right) .
$$

Given the quantities above, we calculate the following average measurements over all branches in generation $i$ :

Average length of branches in generation $g_{i}$ :

$$
\mu_{L}\left(g_{i}\right)=\frac{\sum_{j=p_{i}}^{p_{i}+N^{\mathrm{b}}(i)-1} L(j)}{N^{\mathrm{b}}(i)},
$$

Average CSA of a cross-section in a generation $g_{i}$ branch:

$$
\mu_{A}\left(g_{i}\right)=\frac{\sum_{j=p_{i}}^{p_{i}+N^{\mathrm{b}}(i)-1} \mu_{A}\left(b_{j}\right) N^{\mathrm{c}}(j)}{\sum_{j=p_{i}}^{p_{i}+N^{\mathrm{b}}(i)-1} N^{\mathrm{c}}(j)},
$$

Similar to $\mu_{A}\left(g_{i}\right)$ :

$$
\mu_{\bar{A}}\left(g_{i}\right)=\frac{\sum_{j=p_{i}}^{p_{i}+N^{\mathrm{b}}(i)-1} \mu_{A}\left(b_{j}\right)}{N^{\mathrm{b}}(i)},
$$

but it does not weigh in terms of branch length 
Average distance of a generation $g_{i}$ branch from the root:

$$
\mu_{R}\left(g_{i}\right)=\frac{\sum_{j=p_{i}}^{p_{i}+N^{\mathrm{b}}(i)-1} R\left(b_{j}\right)}{N^{\mathrm{b}}(i)},
$$

Average surface area of a branch in $g_{i}$ :

$$
\mu_{S}\left(g_{i}\right)=\frac{\sum_{j=p_{i}}^{p_{i}+N^{\mathrm{b}}(i)-1} S\left(b_{j}\right)}{N^{\mathrm{b}}(i)},
$$

Average volume of a branch in $g_{i}$ :

$$
\mu_{V}\left(g_{i}\right)=\frac{\sum_{j=p_{i}}^{p_{i}+N^{\mathrm{b}}(i)-1} V\left(b_{j}\right)}{N^{\mathrm{b}}(i)} .
$$

Corresponding standard deviation measurements, $\sigma_{L}\left(g_{i}\right), \sigma_{A}\left(g_{i}\right), \sigma_{\bar{A}}\left(g_{i}\right), \sigma_{R}\left(g_{i}\right), \sigma_{S}\left(g_{i}\right)$, and $\sigma_{V}\left(g_{i}\right)$, are also calculated. (Without loss of generality, we assume branches of interest for each generation have consecutive IDs.)

The volume loss as one traverses a tree is given by

$$
1-V_{\text {loss }}=1-\left(\frac{D_{\mathrm{d} 1}^{3}}{D_{\mathrm{m}}^{3}}+\frac{D_{\mathrm{d} 2}^{3}}{D_{\mathrm{m}}^{3}}\right)=a,
$$

where $D_{\mathrm{d} 1}$ and $D_{\mathrm{d} 2}$ are the lumen diameters of the daughter branches for a mother branch having diameter $D_{\mathrm{m}}$. If all considered branches have circular cross-sections, then the volume of blood available from the mother is proportional to $\frac{1}{6} \pi D_{\mathrm{m}}^{3}$. The volume of blood perfusing through the two daughter branches is in turn proportional to $\frac{1}{6} \pi\left(D_{\mathrm{d} 1}^{3}+D_{\mathrm{d} 2}^{3}\right)$. If the perfusion is "perfect", then

$$
\frac{1}{6} \pi D_{\mathrm{m}}^{3}=\frac{1}{6} \pi\left(D_{\mathrm{d} 1}^{3}+D_{\mathrm{d} 2}^{3}\right) \quad \text { or } \quad 1=\frac{D_{\mathrm{d} 1}^{3}}{D_{\mathrm{m}}^{3}}+\frac{D_{\mathrm{d} 2}^{3}}{D_{\mathrm{m}}^{3}} .
$$

Finally, we also calculate the branching angles between each pair of connected branches. The branching angles of both daughter branches of a mother can be obtained by calculating the enclosing angles between the line segments of the two daughters. This angle $\theta$ is given by Eq. (6), where $\vec{a}$ represents the vector of the segment that approximates the beginning (where the daughter emits from its mother branch) of daughter branch $\# 1$ and $\vec{b}$ is the vector of the segment that approximates the beginning of daughter branch $\# 2$.

$$
\theta=\cos ^{-1}\left(\frac{\vec{a} \cdot \vec{b}}{|\vec{a}||\vec{b}|}\right)
$$

\subsection{Interactive system}

The quantitative data extracted from a typical 3D micro-CT is too overwhelming to use productively without some form of interactive aide. To facilitate interaction with the extracted vasculature and calculated geometrical information, we have designed a visualization system designed for interacting with large micro-CT images depicting tree-like structures. We implemented the system in 

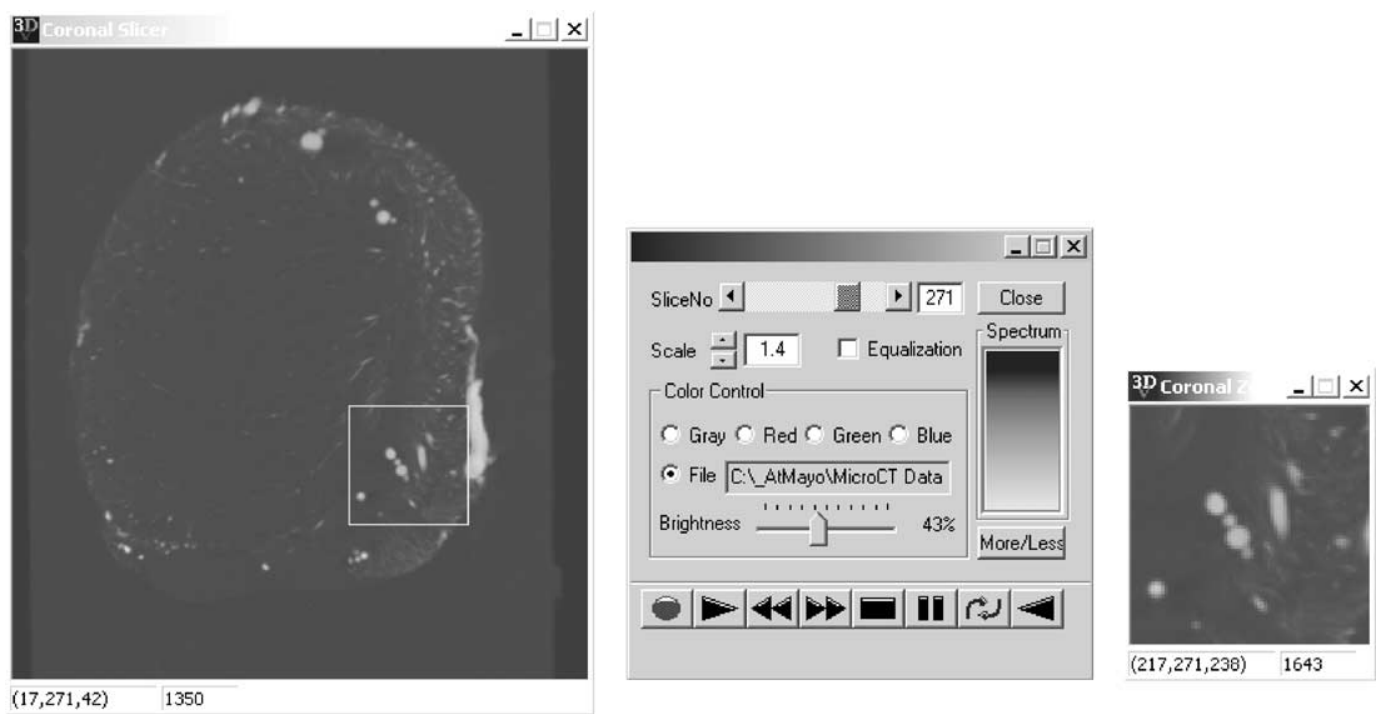

Fig. 5. Example of Coronal Slice Tool with view controls. Coronal slice $(y=271)$ of a $348 \times 348 \times 381$ rat heart image depicted (image 'rsz42'; $\Delta=4.2 \mu \mathrm{m}$ ). Small bright blobs are 2D artery cross-sections. White box on slice is shown magnified in far right view. Transverse and sagittal slice tools also are available.

the Windows 2000 environment, employing Visual $\mathrm{C}++6.0$ and the Microsoft Foundation Class (MFC) framework. For standard graphics functions, we drew upon the Visualization Toolkit (VTK) and OpenGL [20,21]. The system consists of seven visualization tools: (a) three tools for viewing orthographic 2D slices, (b) three tools for viewing orthographic projections, and (c) a multi-mode $3 \mathrm{D}$ tree tool.

Three tools give multi-planar reformatted 2D slice views (i.e., transverse, coronal, sagittal), as shown in Fig. 5. These tools have several capabilities: (a) manipulation of the color scale, brightness, and size of the viewed data; (b) magnification (zooming) in on selected regions; (c) movie controls to view a volume of slices as a movie sequence; (d) statistics and histogram calculation on selected regions; (e) plotting the gray-scale values along a selected line. Three other tools enable the viewing orthogonal projection views of a volume, much like a human chest angiogram (Fig. 6). While a projection can give a misleading impression of the true $3 \mathrm{D}$ geometry of the tree, it does give a useful 2D rendition of the complete data set [18].

The primary interactive tool in the system is the 3D tree tool. It provides a multi-mode facility for visualizing the extracted tree in $3 \mathrm{D}$ and for viewing and correcting the precomputed quantitative data. Figs. 7 and 8 give examples of using this tool. To begin, the segmented image, skeleton representation, and all quantitative data are first loaded. A standard surface-polygon calculation is then done on the segmented data and on the extracted skeleton to generate a representation suitable for rapid interactive surface rendering [21].

At this point, a main interactive window pops up that shows four sub-windows (Fig. 7). In the upper left is the rendering sub-window, where surface rendering and further viewing interactions happen. The lower left sub-window shows text logs when the user interacts with the rendering sub-window. The upper right sub-window controls the viewing effects. The user may adjust the colors, 

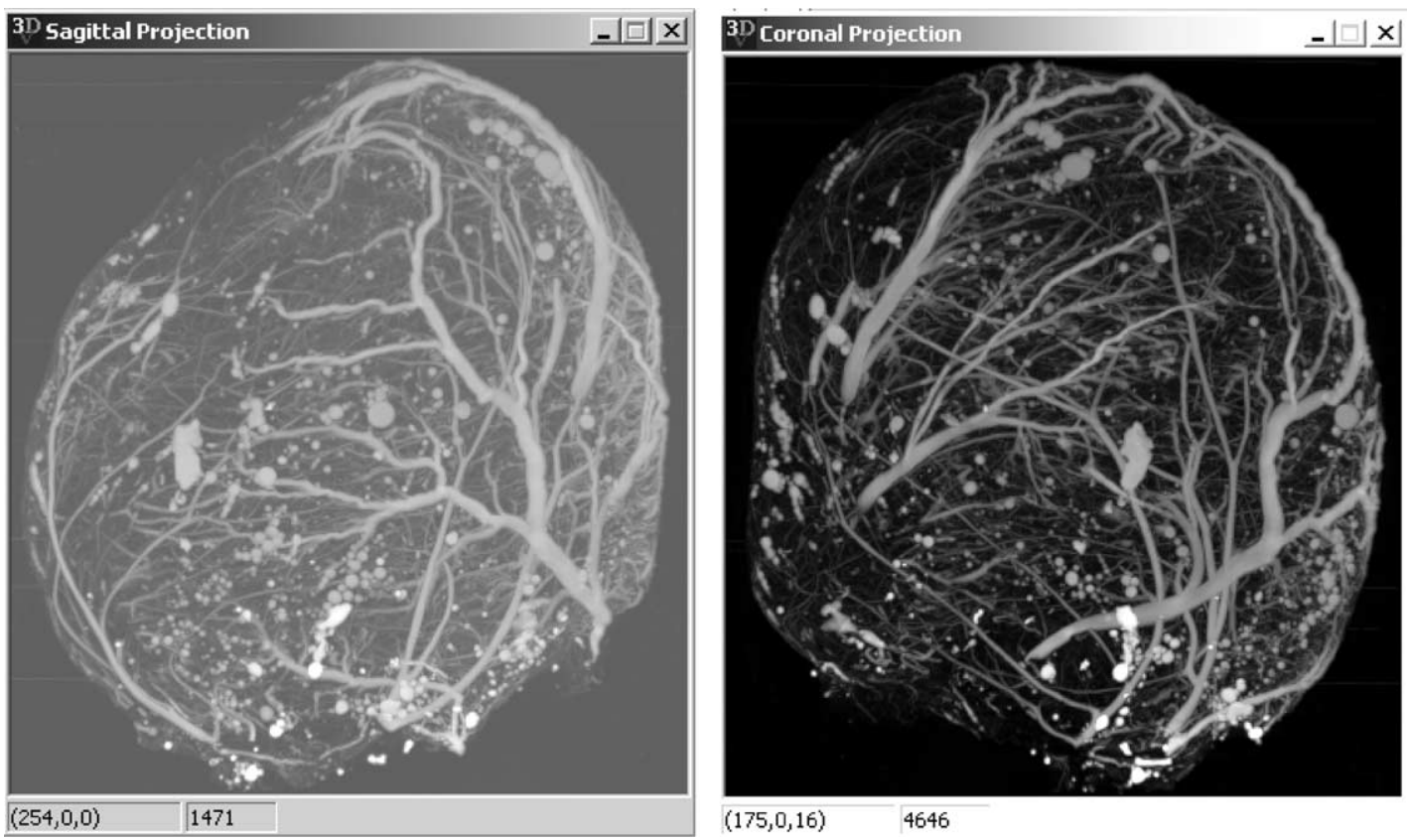

Fig. 6. Examples of sagittal and coronal projection tools. Same image as Fig. 5 used. Maximum intensity projection of entire volume along the $x$-axis (sagittal) and $y$-axis (coronal) depicted. The entire collection of opacified arteries show up in these projection images.

illumination, opacity, thickness, and interaction mode of the objects in the rendering sub-window. In the lower right is the editing sub-window.

There are two operation modes for the 3D tree tool: VTK mode and manual mode. In VTK mode, the left-mouse button is used to rotate the scene. How the scene is rotated depends on where the mouse tip is located. The right-mouse button is used to zoom in and out of the scene. If the location of the mouse tip is in the upper portion of the rendering sub-window when the user clicks on the right-mouse button, then the scene is scaled up; otherwise, if the mouse tip is in the lower portion when the user clicks the rendering sub-window, the scene is scaled down. These are standard graphics interactions, per the VTK package [21].

On the other hand, if the tool is in manual mode, the right-mouse button is disabled, while the left-mouse button is used to "pick" a point on the branching structure in the rendering sub-window. When the user is in the manual mode and clicks on a valid location of the vasculature, the corresponding geometrical and statistical information will appear in the middle of the editing sub-window in real time. It also allows the user to prune branches from the existing vasculature and automatically update the statistical and geometrical information immediately in the text window. This enables manual "on-the-fly" correction of automatically computed results. Fig. 8 shows an editing example where the user interactively pruned a branch and its descendants. The displayed tree is then updated, as are all calculations pertinent to this new form of the tree. Note that this "recalculation" is very quick, as all computations had already been done; branch pruning merely involves deleting certain branches from the precomputed representation and updating the affected generations. 


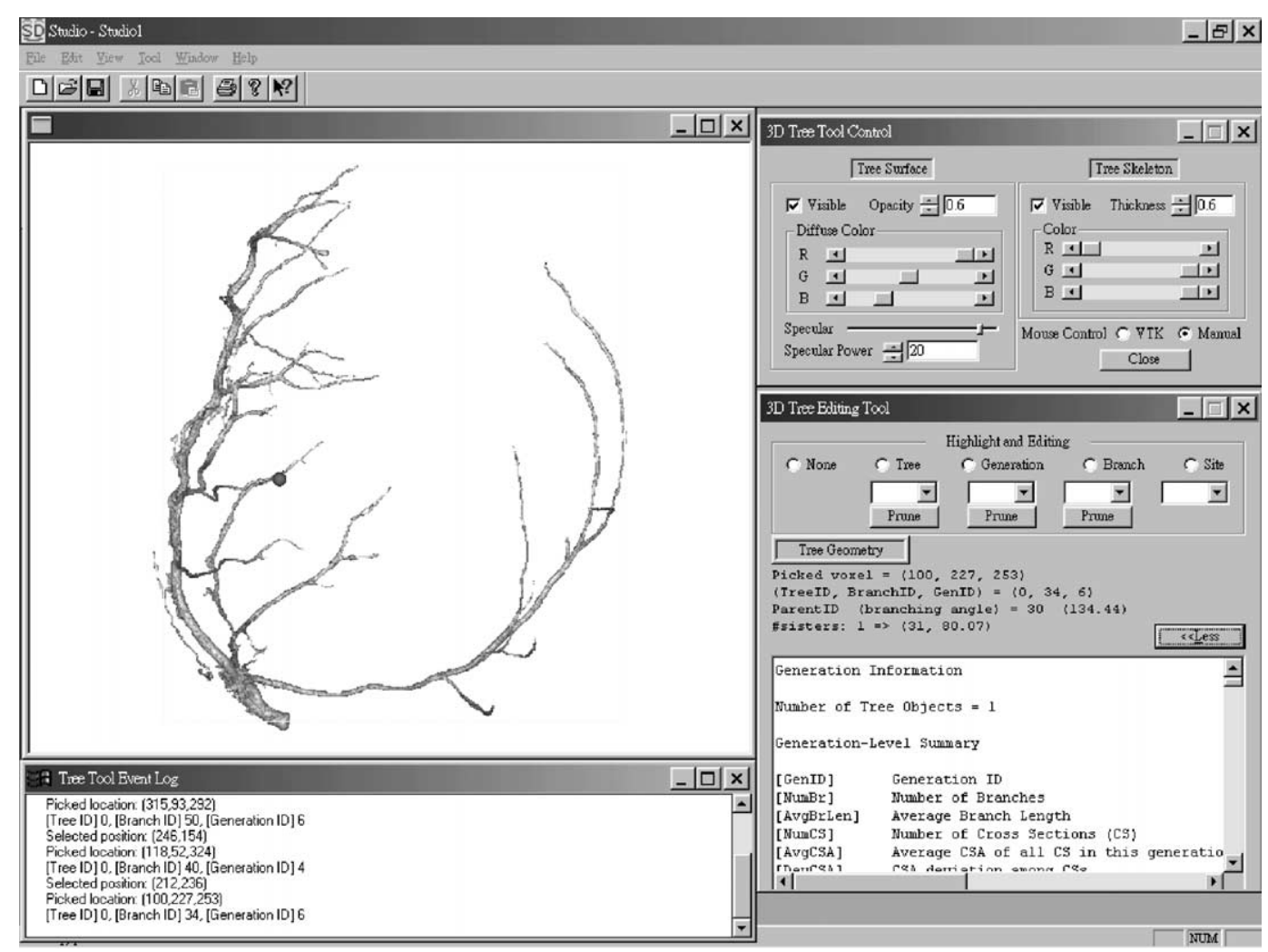

Fig. 7. 3D tree tool and its controls. Image lca_146 used. The upper left is the viewing window showing the surface rendering of the segmented tree that is overlaid with the extracted skeleton. The upper right panel governs the control of the tool and rendering properties. The lower right depicts the 3D tree-editing tool. The bottom left presents further information detailing the interactive session.

\section{Results}

Figs. 9-11 illustrates the raw quantitative information extracted for a typical micro-CT rat heart image. The final computed quantitative data consists of information present at three hierarchical levels:

- Generation level: an overall statistical summary of the characteristics of each generation.

- Inter-branch level: an overall summary of each branch.

- Intra-branch level: a detailed description of the data for each branch.

The presented example gives an impression of the nature and exhaustiveness of the information computed. Per Fig. 9, generation $g_{i}=2$ has four branches and its average branch length $\mu_{L}\left(g_{i}\right)=$ $639.6 \mu \mathrm{m}$, etc. (Note that we use $10 \mu \mathrm{m}$ as a measurement unit in the tables.) By observation from the generation-level information, we can begin to grasp an overall understanding of the vasculature's properties. Fig. 10 gives a complete breakdown of the average properties of every individual extracted branch in the tree. Finally, Fig. 11 gives an excerpted breakdown of all acquired data for a particular 


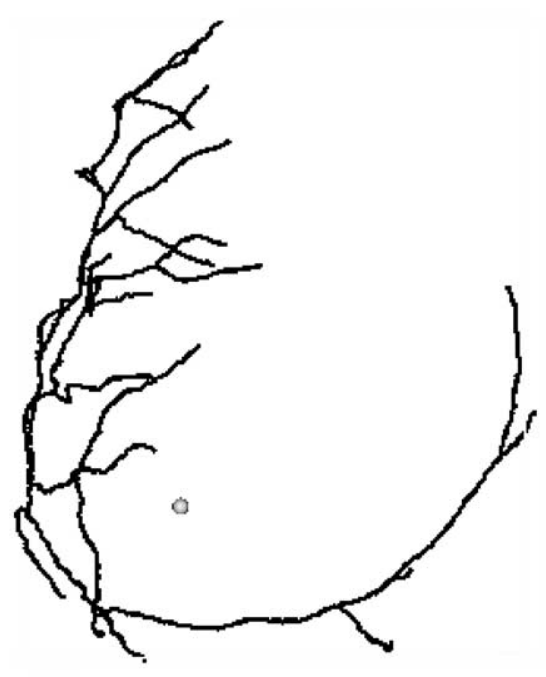

(a) Extracted tree skeleton of lca_146

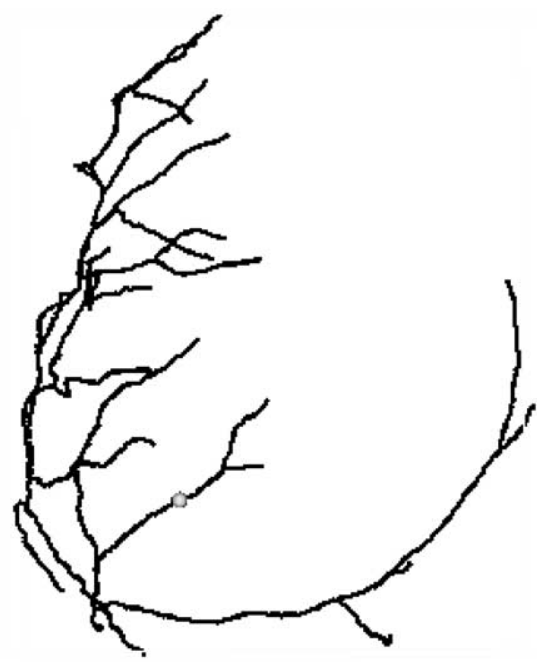

(b) Edited tree

Fig. 8. Editing on the extracted tree. Part (a) illustrates the extracted 3D tree skeleton of lca_146. The user places a sphere on certain location indicating the branch and beyond are of interest. (b) Shows the pruning result. As it is a $3 \mathrm{D}$ editing, the user may need to rotate or scale the tree skeleton to find an appropriate viewing direction to place the sphere.

\begin{tabular}{|c|c|c|c|c|c|c|c|c|c|c|c|c|c|}
\hline$g_{i}$ & $N^{b}(i)$ & $\mu_{L}\left(g_{i}\right)$ & $N^{c}\left(g_{i}\right)$ & $\mu_{A}\left(g_{i}\right)$ & $\sigma_{A}\left(g_{i}\right)$ & $\mu_{\bar{A}} g_{i}$ & $\sigma_{\bar{A}}\left(g_{i}\right)$ & $\mu_{S}\left(g_{i}\right)$ & $\sigma_{S}\left(g_{i}\right)$ & $\mu_{V}\left(g_{i}\right)$ & $\sigma_{V}\left(g_{i}\right)$ & $\mu_{D}\left(g_{i}\right)$ & $\sigma_{D}\left(g_{i}\right)$ \\
\hline 0 & 1 & 89.44 & 56 & 835.61 & 288.79 & 835.61 & 0.00 & 11781.54 & 0.00 & 97332.22 & 0.00 & 0.00 & 0.00 \\
\hline 1 & 2 & 145.60 & 176 & 240.19 & 481.45 & 549.48 & 327.92 & 9436.71 & 8347.97 & 43964.18 & 34839.28 & 128.29 & 0.00 \\
\hline 2 & 4 & 63.96 & 161 & 302.31 & 511.03 & 461.78 & 365.59 & 4478.58 & 1512.95 & 25309.44 & 12077.86 & 312.92 & 174.58 \\
\hline 3 & 6 & 170.21 & 684 & 180.58 & 262.91 & 253.34 & 189.15 & 10168.22 & 4721.62 & 42819.82 & 24040.97 & 314.36 & 164.24 \\
\hline 4 & 6 & 101.57 & 381 & 138.27 & 232.08 & 190.54 & 199.56 & 4930.35 & 2522.73 & 18263.29 & 10268.31 & 585.35 & 182.87 \\
\hline 5 & 2 & 158.08 & 199 & 96.88 & 685.01 & 561.13 & 483.70 & 5808.30 & 4855.70 & 20049.57 & 11356.63 & 432.17 & 0.00 \\
\hline 6 & 2 & 147.68 & 187 & 334.80 & 263.68 & 330.58 & 112.63 & 11955.29 & 2563.66 & 65111.62 & 24310.56 & 438.41 & 0.00 \\
\hline 7 & 4 & 134.68 & 351 & 145.79 & 154.80 & 164.68 & 100.33 & 7007.98 & 1255.14 & 26609.78 & 12390.09 & 636.14 & 12.68 \\
\hline 8 & 4 & 55.12 & 137 & 134.75 & 186.37 & 206.43 & 122.24 & 2686.13 & 1419.14 & 9599.59 & 5293.33 & 793.35 & 5.78 \\
\hline 9 & 2 & 42.64 & 53 & 367.50 & 175.26 & 347.95 & 60.96 & 3652.67 & 1502.62 & 20256.55 & 9511.85 & 790.52 & 0.00 \\
\hline 10 & 4 & 52.52 & 131 & 188.81 & 159.77 & 203.62 & 98.70 & 3066.42 & 727.87 & 12861.69 & 5112.99 & 846.14 & 19.50 \\
\hline 11 & 6 & 118.91 & 461 & 72.02 & 95.53 & 91.95 & 68.35 & 4343.45 & 837.79 & 11509.61 & 2980.78 & 921.12 & 7.96 \\
\hline 12 & 2 & 107.12 & 131 & 101.19 & 95.24 & 125.95 & 75.42 & 4379.99 & 202.94 & 13786.33 & 4643.44 & 992.42 & 0.00 \\
\hline 13 & 2 & 108.16 & 137 & 123.98 & 101.26 & 130.75 & 40.33 & $\begin{array}{l}5158.79 \\
\end{array}$ & 304.76 & 17664.86 & 2618.68 & 1082.76 & 0.00 \\
\hline 14 & 2 & 108.16 & 143 & 62.90 & 34.04 & 62.47 & 2.90 & 4051.40 & $\begin{array}{l}673.32 \\
\end{array}$ & 9354.37 & 1795.28 & 1210.08 & 0.00 \\
\hline
\end{tabular}

Fig. 9. Example summary at the generation level. The results are from the micro-CT rat image lca_146. Note that in order to compress the results, the unit measure used for length is "10 $\mu \mathrm{m}$ "; area " $(10 \mu \mathrm{m})^{2}$ "; volume " $(10 \mu \mathrm{m})^{3}$ ".

branch. In our experience, these data tend to be too detailed for interactive study, but they are an essential data element necessary for computing the average branch-level and generation-level information.

While much useful information exists in these analysis, Fig. 9 points out problems in the analysis. At generation 3, we would expect there to be eight branches, instead of six. Also, subsequent generations indicate similar inconsistencies on the number of branches per generation. Generations consisting of the smallest branches appear to have many missing branches. Data imperfections, such as partial-volume artifacts, point-spread function blurring, and specimen preparation, contribute to 


\begin{tabular}{|c|c|c|c|c|c|c|c|c|c|c|c|}
\hline$g_{i}$ & $j$ & $P_{I D}\left(b_{j}\right)$ & $L\left(b_{j}\right)$ & $N^{c}(j)$ & $\mu_{A}\left(b_{j}\right)$ & $\sigma_{A}\left(b_{j}\right)$ & $L D\left(b_{j}\right)$ & $S\left(b_{j}\right)$ & $V\left(b_{j}\right)$ & $D\left(b_{j}\right)$ & $V_{\text {loss }}\left(b_{j}\right)$ \\
\hline \hline 0 & 0 & -1 & 128.29 & 56 & 835.61 & 288.79 & 2.00 & 11781.54 & 97332.22 & 0.00 & -0.23 \\
\hline 1 & 1 & 0 & 10.04 & 5 & 877.39 & 136.55 & 0.76 & 1088.74 & 9124.90 & 128.29 & -0.55 \\
\hline 1 & 42 & 0 & 359.20 & 171 & 221.56 & 196.37 & 3.01 & 17784.69 & 78803.46 & 128.29 & -0.28 \\
\hline 2 & 2 & 1 & 32.42 & 15 & 1040.07 & 565.00 & 1.30 & 3431.24 & 32450.07 & 138.34 & 0.29 \\
\hline 2 & 37 & 1 & 89.73 & 39 & 505.63 & 281.66 & 1.96 & 6208.19 & 41017.04 & 138.34 & 0.84 \\
\hline 2 & 43 & 42 & 56.77 & 25 & 199.36 & 50.20 & 1.94 & 2582.65 & 10366.75 & 487.50 & -0.08 \\
\hline 2 & 48 & 42 & 177.96 & 82 & 102.04 & 84.76 & 2.95 & 5692.25 & 17403.89 & 487.50 & 1.00 \\
\hline 3 & 3 & 2 & 209.11 & 99 & 441.95 & 130.16 & 2.42 & 15198.65 & 91005.99 & 170.75 & -0.82 \\
\hline 3 & 36 & 2 & 58.29 & 26 & 583.73 & 337.84 & 1.70 & 4407.62 & 31568.18 & 170.75 & 1.00 \\
\hline 3 & 38 & 37 & 481.51 & 229 & 91.40 & 88.81 & 3.70 & 14729.50 & 43536.73 & 228.07 & 1.00 \\
\hline 3 & 39 & 37 & 324.04 & 155 & 109.14 & 90.08 & 3.29 & 11334.23 & 35185.74 & 228.07 & -0.49 \\
\hline 3 & 44 & 43 & 279.82 & 133 & 158.26 & 51.98 & 2.98 & 12176.45 & 43779.70 & 544.26 & -0.04 \\
\hline 3 & 47 & 43 & 88.01 & 42 & 135.56 & 136.08 & 2.42 & 3162.88 & 11842.57 & 544.26 & 1.00 \\
\hline 4 & 4 & 3 & 52.30 & 24 & 635.44 & 164.47 & 1.61 & 4426.73 & 31721.16 & 379.87 & -1.18 \\
\hline 4 & 35 & 3 & 168.42 & 82 & 115.12 & 141.74 & 2.92 & 5631.24 & 19635.62 & 379.87 & 1.00 \\
\hline 4 & 40 & 39 & 52.61 & 29 & 110.25 & 77.78 & 2.10 & 2132.62 & 6650.20 & 552.11 & 1.00 \\
\hline 4 & 41 & 39 & 127.74 & 63 & 74.58 & 99.12 & 2.92 & 3594.13 & 9772.82 & 552.11 & 1.00 \\
\hline 4 & 45 & 44 & 257.18 & 128 & 118.67 & 55.22 & 3.07 & 10067.19 & 31595.18 & 824.08 & 1.00 \\
\hline 4 & 46 & 44 & 115.36 & 55 & 89.20 & 41.98 & 2.68 & 3730.20 & 10204.77 & 824.08 & 1.00 \\
\hline 5 & 5 & 4 & 6.24 & 4 & 1044.83 & 82.46 & 0.52 & 952.59 & 8692.95 & 432.17 & 0.67 \\
\hline 5 & 34 & 4 & 399.11 & 195 & 77.43 & 180.84 & 3.78 & 10664.00 & 31406.20 & 432.17 & 1.00 \\
\hline 6 & 6 & 5 & 210.41 & 97 & 443.21 & 296.05 & 2.44 & 14518.95 & 89422.18 & 438.41 & 0.20 \\
\hline 6 & 29 & 5 & 185.05 & 90 & 217.95 & 161.24 & 2.62 & 9391.63 & 40801.06 & 438.41 & 0.35 \\
\hline
\end{tabular}

Fig. 10. Example summary at the branch level. The results are from the micro-CT rat image lca_146. The unit measure used for length is " $10 \mu \mathrm{m}$ ", for area " $(10 \mu \mathrm{m})^{2}$ ", and for volume " $(10 \mu \mathrm{m})^{3 "}$. $P_{\mathrm{ID}}\left(b_{j}\right)$ gives the parent branch ID for $b_{j}$. For brevity, information for generations 7 through 14 is omitted.

\begin{tabular}{|c|c|c|c|c|}
\hline Branch ID $(j)$ & Generation ID $(i)$ & Number of Voxels & Distance to Root $\left(D\left(b_{j}\right)\right)$ & Parent ID and branching angle \\
\hline 0 & 0 & 43 & 0.00 & NULL (N/A) \\
\hline $\begin{array}{r}\text { Sisters: } \\
\text { Daughters: } \\
\text { CSAs: }\end{array}$ & 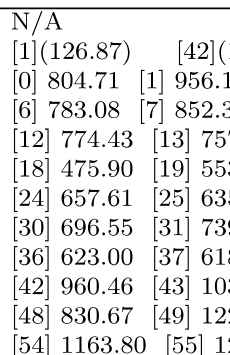 & 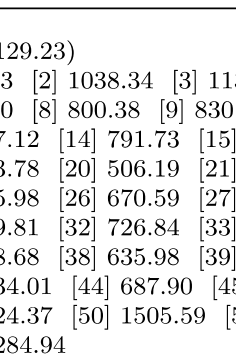 & 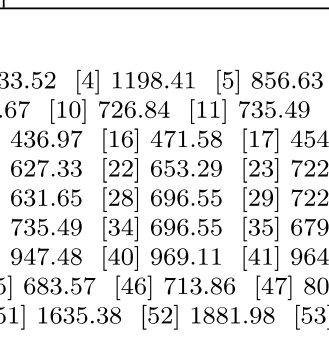 & $\begin{array}{l}27 \\
51 \\
51 \\
24 \\
79 \\
9.04 \\
1388.77\end{array}$ \\
\hline
\end{tabular}

Fig. 11. Example detailed information for a particular branch. The results are from the micro-CT rat image lca_146. The unit measure used for length is " $10 \mu \mathrm{m}$ "; area " $(10 \mu \mathrm{m})$ "; volume " $(10 \mu \mathrm{m})^{3}$ "; angle "degree".

these inconsistencies. Regarding specimen preparation, bubbles arising from air or imperfect penetration of the contrast agent) can "break" branches and alter the overall computed tree geometry. Also, poor contrast filling of small distal vessels will cause many vessels not to appear. Further work in improving specimen preparation, scan quality, and computer processing will ameliorate these factors. Yet, much useful data can be gleaned from the current analysis procedure. 


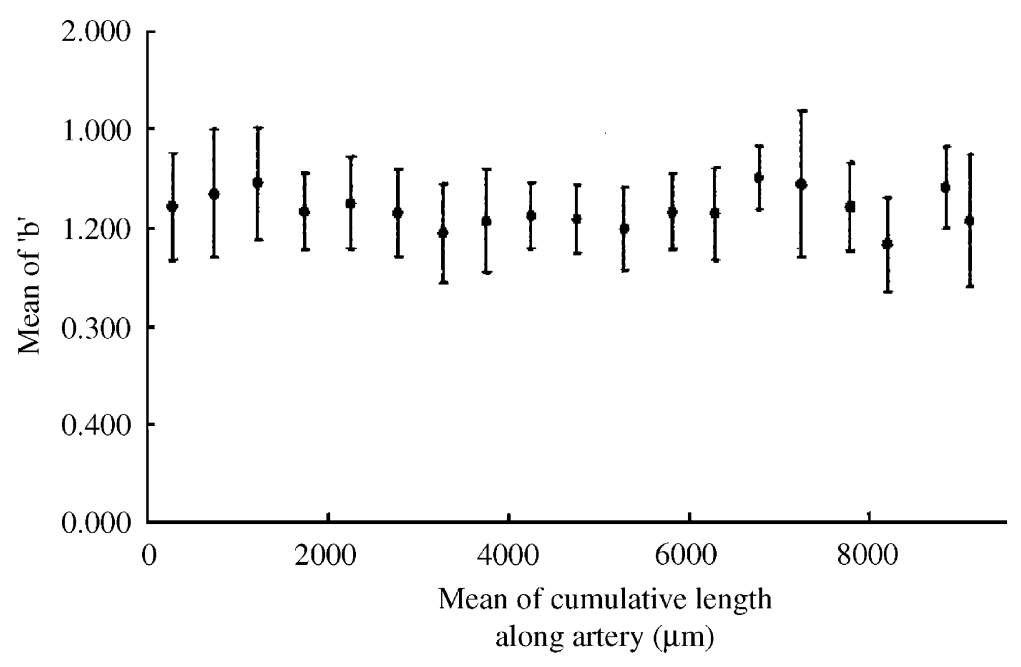

Fig. 12. The value of $b$ in the relation $L=c D^{b}$ between arterial inter-branch segmental length $(L)$ and diameter $(D)$ [22].

To illustrate, results are presented for a hypothesized relationship between the arterial inter-branch segmental length and diameter [6]:

$$
L=c D^{b},
$$

where $D$ is the diameter of an arterial, inter-branch segment of length $L$ and $c$ and $b$ are constants. Quantities $D$ and $L$ are as defined in Section 3.1, but with the branch ID $b_{j}$ suppressed for brevity. Eq. (7) has no obvious physiological basis other than it has been shown to occur. Fig. 12 is a plot of $b$ as it varies along an artery's length from the aortic origin to the smallest vessel analyzed, which, in our case, is less than $50 \mu \mathrm{m}$ in diameter (two voxels). Note that the variation between the branches of the same branching order remains essentially unchanged along the length of the vessel. We again caution that data imperfections could result in missing or corrupted branches that could adversely affect these analyses.

\section{Discussion}

The proposed methodology has enabled us to validate both the method of Ref. [4] and the quantitative analyses of this paper. Our current implementation employs line segments to approximate branches and to calculate cross-sectional areas. Further research will be on smoothed branch approximations to obtain more robust quantitative analysis results. A database housing approach would be beneficial to help archive and retrieve the overwhelming amount of generated analyses results. The incorporation of various charting methods may also help visualize the quantitative data in different aspects.

While the quantitative results support the arguments in Refs. [22-25], considerable work is needed in understanding whether imperfections are attributable to data collection and analysis, "noise" (heterogeneity) in the branching structure [22], or to fluid-dynamic consequences of the branching structure. It is important to realize that our methods have only been definitively validated for one complete 
branch. While our results to date for performing a true exhaustive multi-generational analysis are encouraging, we do know that the aforementioned imperfections can cause either small missing branches, breaks in branches, or loops (which are not physiologically plausible). These imperfections can cause certain branches to be missed and to result in an incorrect branch ordering. Definitive multi-generational ground-truth data under real scanning circumstances is needed to understand the impact of incorrect ordering. An important task to do to address this issue is to expand the visualization tools, so that manual or semi-automatic visual editing methods are introduced to correct missing branches, breaks, and loops.

\section{Summary}

Modern micro-CT scanners can produce large high-resolution three-dimensional (3D) digital images of vascular trees. A typical tree consists of hundreds of branches and many generations. The spatial resolution of $3 \mathrm{D}$ micro-CT images is fine enough to permit detailed real studies of basic physiologic questions on the coronary arterial tree. Performing a study requires three steps: (1) extract the arterial from the image; (2) compute quantitative data from the extracted tree; and (3) perform a numerical analysis of the computed data. Because of the complexity of the coronary arterial tree, it is impractical to perform such an integrated study manually. An automatic method exists for performing step (1), extracting the tree, but no effort has been made on the other two steps.

We propose an environment for performing a complete study. A set of quantitative statistical measures are discussed. Information on cross-sectional area, branch length, branch surface area and other measures at the generation, inter-branch, and intra-branch levels are computed. A human user can then work with the quantitative data in an interactive visualization system. The system provides various forms of $2 \mathrm{D}$ and $3 \mathrm{D}$ viewing, such as multiplanar reformatted slices, projection images, and surface renderings. It also permits interactive tree editing for modifying the extracted tree and correct the computed quantitative data.

Results are given that illustrate the three levels of quantitative data computed. Also, the methodology is applied to testing the validity of a hypothesized physiologic law for the arterial tree.

\section{Acknowledgements}

This work was partially supported by National Science Council, Taiwan, ROC through grant NSC-89-2314-B-182-147-M08, by NIH grants R01-RR11800 and R01-CA74325, by the Whitaker Foundation, and by NSF grant BIR-9317816 under the Instrument Development for Biological Research Program. Patricia Lund helped prepare the specimens and Steven Jorgensen scanned the specimens.

\section{References}

[1] R.H. Johnson, H. Hu, S.T. Haworth, P.S. Cho, C.A. Dawson, J.H. Linehan, Feldkamp and circle-and-line conebeam reconstruction for 3D micro-CT of vascular networks, Phys. Med. Biol. 43 (4) (1998) 929-940.

[2] S.M. Jorgensen, O. Demirkaya, E.L. Ritman, Three dimensional imaging of vasculature and parenchyma in intact rodent organs with X-ray micro-CT, Am. J. Physiol. Heart. Circ. Physiol. 44275 (1998) H1103-H1114. 
[3] M.J. Paulus, H. Sari-Sarraf, S.S. Gleason, M. Bobrek, J.S. Hicks, D.K. Johnson, A new X-ray computed tomography system for laboratory mouse imaging, IEEE Trans. Nucl. Sci. 46 (3) (1999) 558-564.

[4] Shu-Yen Wan, Atilla Kiraly, Erik L. Ritman, William Higgins, Extraction of the hepatic vasculature in rats using 3D micro-CT images, IEEE Trans. Med. Imaging 19 (9) (2000) 964-971.

[5] N. MacDonald, Trees and Networks in Biological Models, Wiley, New York, 1983.

[6] K. Suwa, T. Niwa, H. Fukusawa, Y. Sasaki, Estimation of intravascular blood pressure by mathematical analysis of arterial casts, Tohoku J. Exp. Med. 79 (1963) 168-198.

[7] T.M. Griffith, D.W. Edwards, Basal EDRF activity helps to keep the geometrical configuration of arterial bifurcations close to the Murray optimum, J. Theor. Biol. 146 (1990) 545-573.

[8] C.D. Murray, The physiological principle of minimum work i. The vascular system and the cost of blood volume, in Proc. Nat. Acad. Sci. USA, 1926, Vol. 12, pp. 207-214.

[9] C.D. Murray, The physiological principle of minimum work applied to the branching of arteries, J. Gen. Physiol. 9 (1926) 835-841.

[10] M. Zamir, Nonsymmetrical bifurcations in arterial branching, J. Gen. Physiol. 72 (1978) 837-845.

[11] Roger H. Johnson, Kelly L. Karau, Robert C. Molthen, Christopher A. Dawson, Exploiting self-similarity of arterial tree branches to reduce the complexity of analysis, in: C.T. Chen, A.V. Clough (Eds.), SPIE Medical Imaging 1999: Physiology and Function from Multidimensional Images, Vol. 3660, 1999, pp. 351-361.

[12] R.H. Johnson, K.L. Karau, R.C. Molthen, S.T. Haworth, C.A. Dawson, Micro-CT image-derived metrics to quantify arterial wall distensibility reduction in a rat model of pulmonary hypertension, in: A. Clough, C.T Chen (Eds.), Proceedings of SPIE Medical Imaging 2000: Physiology and Function from Multidimensional Images, Vol. 3978, 2000.

[13] J.-S. Lee, Digital image smoothing and the sigma filter, Comput. Vis. Graphics Image Processing 24 (1983) $255-269$.

[14] Shu-Yen Wan, William Higgins, Symmetric region growing, Proceedings of IEEE International Conference on Image Processing (ICIP2000), Vol. II, September 10-13, 2000, Vancouver, British Columbia, Canada, pp. 96-99.

[15] P.K. Saha, B.B. Chaudhuri, D. Dutta Majumder, A new shape preserving parallel thinning algorithm for 3D digital images, Pattern Recognition 30 (12) (1997) 1939-1955.

[16] K. Mori, J.-I. Hasegawa, Y. Suenaga, J.-I. Toriwaki, Automated anatomical labeling of the bronchial branch and its application to the virtual bronchoscopy system, IEEE Trans. Med. Imaging 19 (2) (2000) 104-114.

[17] Shu-Yen Wan, Erik L. Ritman, William E. Higgins, Multigenerational analysis and visualization of large 3D vascular images, in: Milan Sonka, Kenneth M. Hanson (Eds.), Proceedings of SPIE Medical Imaging 2001: Image Processing, Vol. 4332, to appear in June 2001.

[18] W.E. Higgins, R.A. Karwoski, W.J.T. Spyra, E.L. Ritman, System for analyzing true three-dimensional angiograms, IEEE Trans. Med. Imaging 15 (3) (1996) 377-385.

[19] Shu-Yen Wan, Analysis and visualization of large branching networks in 3D digital images, Ph.D. Dissertation, Department of Computer Science and Engineering, The Pennsylvania State University, August 2000.

[20] Renate Kempf, Chris Frazier, OpenGL reference manual, Addison Wesley, Reading, MA, 1997.

[21] Will Schroeder, Ken Martin, Bill Lorensen, The Visualization Toolkit, 2nd Edition, Prentice-Hall, Upper Saddle River, NJ, 1998.

[22] Shu-Yen Wan, Patricia E. Lund, Denise A. Reyes, Catherine A. Seaman, William E. Higgins, Erik L. Ritman, Heterogeneity of coronary arterial branching geometry, in: A. Clough, C.T. Chen (Eds.), Proceedings of SPIE Medical Imaging 2000: Physiology and Function from Multidimensional Images, Vol. 3978, 2000, pp. 515-520.

[23] J.B. Bassingthwaighte, R.B. King, S.A. Roger, Fractal nature of regional myocardial blood flow heterogeneity, Circ. Res. 65 (1989) 578-590.

[24] S. Cavalcanti, M. Ursino, Chaotic oscillations in microvessel arterial networks, Ann. Biomed. Eng. 24 (1996) $37-47$.

[25] E. van Bavel, J.A.E. Spaan, Branching patterns in the porcine coronary arterial tree estimation of flow heterogeneity, Circ. Res. 71 (1992) 1200-1212.

Shu-Yen Wan received the B.S. in Computer Science from Chung Yuan Christian University, Taiwan, the M.S. in Computer Science from the National Chung Cheng University, Taiwan, and the Ph.D. in Computer Science and Engineering from Penn State University, 
University Park, PA. He is currently an Assistant Professor in Information Management at the Chang Gung University, Taiwan, R.O.C. His research interests are in the general areas of image processing and visualization, computer vision, and medical imaging.

Erik L. Ritman received the B.Sc. in Physics and the M.D. degree in Medicine and Surgery from Melbourne University, Melbourne, Australia. He has also received the Ph.D. in Physiology from the Mayo Graduate School of Medicine, Rochester, MN. He is currently a Professor of Physiology and Medicine at the Mayo Medical School, Rochester, MN. His research interests are in the general area of 3D cardiopulmonary imaging.

William E. Higgins received the B.S. in Electrical Engineering from MIT and the M.S. and Ph.D. in Electrical Engineering from the University of Illinois. He was a Principal Research Scientist at the Honeywell Systems and Research Center, Minneapolis, MN, and a Research Fellow within the Biodynamics Research Unit of the Mayo Clinic, Rochester, MN. He is currently a Professor of Electrical Engineering at the Pennsylvania State University. He is also an affiliated member of the Computer Science and Engineering Department and the Bioengineering Program at Penn State. In addition he is an adjunct Professor of Radiology at the University of Iowa. His research interests are in multidimensional medical image processing and visualization and in virtual endoscopy. He is a member of IEEE, SPIE, Pattern Recognition Society, Eta Kappu Nu, Tau Beta Pi, and Sigma Xi. 